\title{
Neuroprotective potential of imatinib in global ischemia- reperfusion-induced cerebral injury: possible role of Janus-activated kinase 2/signal transducer and activator of transcription 3 and connexin 43
}

\author{
Jieying Wang ${ }^{1}$, Taomin Bai ${ }^{1}$, Nana Wang ${ }^{2}$, Hongyan $\mathrm{Li}^{1}$, and Xiangyang Guo ${ }^{1,3, *}$ \\ 'Department of Pediatrics and ${ }^{2}$ Central Laboratory, Shaanxi Provincial People's Hospital, The Affiliated Hospital of Xi'an Medical University, The Third Affiliated \\ Hospital of Xi'an Jiaotong University, Xi'an 710068, ${ }^{3}$ Department of Neurology, Xijing Hospital, Air Force Medical University, Xi'an 710032, Shaanxi, China
}

\section{ARTICLE INFO}

Received May 17, 2019

Revised June 18, 2019

Accepted June 20, 2019

\section{*Correspondence \\ Xiangyang Guo \\ E-mail: sxxaped@sina.com}

\section{Key Words}

Connexin 43

Imatinib mesylate

Ischemia

Neuroprotection

Reperfusion

STAT3 transcription factor

\begin{abstract}
The present study was aimed to explore the neuroprotective role of imatinib in global ischemia-reperfusion-induced cerebral injury along with possible mechanisms. Global ischemia was induced in mice by bilateral carotid artery occlusion for $20 \mathrm{~min}$, which was followed by reperfusion for $24 \mathrm{~h}$ by restoring the blood flow to the brain. The extent of cerebral injury was assessed after $24 \mathrm{~h}$ of global ischemia by measuring the locomotor activity (actophotometer test), motor coordination (inclined beam walking test), neurological severity score, learning and memory (object recognition test) and cerebral infarction (triphenyl tetrazolium chloride stain). Ischemia-reperfusion injury produced significant cerebral infarction, impaired the behavioral parameters and decreased the expression of connexin 43 and phosphorylated signal transducer and activator of transcription 3 (p-STAT3) in the brain. A single dose administration of imatinib (20 and $40 \mathrm{mg} / \mathrm{kg}$ ) attenuated ischemia-reperfusioninduced behavioral deficits and the extent of cerebral infarction along with the restoration of connexin 43 and p-STAT3 levels. However, administration of AG490, a selective Janus-activated kinase 2 (JAK2)/STAT3 inhibitor, abolished the neuroprotective actions of imatinib and decreased the expression of connexin 43 and p-STAT3. It is concluded that imatinib has the potential of attenuating global ischemia-reperfusion-induced cerebral injury, which may be possibly attributed to activation of JAK2/ STAT3 signaling pathway along with the increase in the expression of connexin 43 .
\end{abstract}

\section{INTRODUCTION}

Imatinib is a selective tyrosine kinase inhibitor and it is widely used as an anticancer drug primarily in Philadelphia-chromosome-positive chronic myeloid leukemia and gastrointestinal stromal tumor $[1,2]$. Besides its well documented anticancer actions, scientists have identified its therapeutic potential in diseases affecting the brain such as Alzheimer disease, Parkinson disease, multiple stroke and brain hemorrhage etc. [3-5]. More- over, its potential in preventing the damage to the blood brain barrier in different animal models has been reported [6-10]. However, its role and mechanisms in global cerebral ischemiareperfusion-induced impairment in motor activity, learning and memory deficits and cerebral infarction is not explored. A state of global cerebral ischemia-reperfusion injury in rodents mimics the situation of severe shock in patients due to cardiac arrest or severe hypotension [11,12]. Accordingly, a rodent model of global ischemia reperfusion-induced injury was employed to explore the (i) (8) This is an Open Access article distributed under the terms of the Creative Commons Attribution Non-Commercial License, which permits unrestricted non-commercial use, distribution, and reproduction in any medium, provided the original work is properly cited. Copyright $\odot$ Korean J Physiol Pharmacol, pISSN 1226-4512, elSSN 2093-3827
Author contributions: J.W. performed the experiments. T.B. helped in performing experiments. N.W. analyzed the data. H.L. wrote the manuscript. X.G. conceived the idea and did the editing part. 
neuroprotective role of imatinib.

Janus-activated kinase 2/signal transducer and activator of transcription 3 (JAK2/STAT3) constitute an important signaling pathway in the brain. Indeed, the activation of JAK2 leads to phosphorylation of STAT3, which is translocated to nucleus to serve as a transcriptional factor. Studies have reported the significant role of JAK2/STAT3 in the different brain diseases, particularly in ischemic brain disease. The role of JAK2/STAT3 in ischemic brain has been delineated by using alpha-cyano-(3,4dihydroxy)-N-benzylcinnamide (AG490) as a selective JAK2/ STAT3 inhibitor [13-15]. Gap junctions are the intercellular channels and these allow the communication between the adjacent cells. Connexin 43 is an important constituent protein of the gap junction channels and connexin 43 is widely distributed in the central nervous system including astrocytes, neuronal precursors and neurons $[16,17]$. Moreover, studies have reported the key role of connexin 43 in cerebral injury and accordingly, scientists have projected connexin 43 as a key therapeutic target in inducing tissue protection [18-20]. The present study was aimed to explore the neuroprotective role of imatinib in global ischemia-reperfusioninduced cerebral injury along with possible mechanisms involving JAK3/STAT3 and connexin 43.

\section{METHODS}

\section{Experimental animals, chemicals and drugs}

Swiss albino male mice (20-25 g) were used for this study. The experimental studies pertaining to animals were approved by Animal Ethics Committee of Shaanxi Provincial People's Hospital (no. 201905-1301) and all experiments were done according to ethical guidelines. The enzyme-linked immunosorbent assay (ELISA) kit for the quantitative estimation of phosphorylated (p)STAT3 was procured from Abcam, Cambridge, MA, USA, while the ELISA kit for connexin 43 was procured from LifeSpan BioSciences, Seattle, WA, USA. Imatinib mesylate (Sigma Aldrich, St. Louis, MO, USA) was dissolved in normal saline, while AG490 (Sigma Aldrich) was dissolved in 2\% dimethyl sulfoxide and their doses were selected on the basis of previously published reports [21-24].

\section{Global ischemia reperfusion injury to mice brain}

Thiopental sodium (45 mg/kg i.p.) was administered to anesthetize mice. Thereafter, surgery was performed to occlude both carotid arteries for $20 \mathrm{~min}$ and induce global ischemic injury. It was followed by reperfusion injury in which occlusion was removed and the blood flow to the brain was restored for $24 \mathrm{~h}$ $[25,26]$.

\section{Behavioral assessment of extent of ischemia- reperfusion-induced cerebral injury}

The extent of ischemia-reperfusion injury was assessed by using a battery of behavioral tests to evaluate the locomotion, motor coordination and cognition. The locomotor activity was assessed using an actophotometer test for $10 \mathrm{~min}$ and activity was recorded in scores. The inclined beam walking test was used to evaluate the fore- and hind limb motor coordination in which mice were allowed to walk on an inclined (at an angle of $60^{\circ}$ ) metallic bar of $60 \mathrm{~cm}$ length and $1.5 \mathrm{~cm}$ width. The scoring between $0-4$ was done on the basis of their performance on the metallic, with the lowest score indicating no defect and highest score indicating the maximum defect. The total neurological deficit was also assessed by giving the scores from $0-10$, with lowest score indicating the normal and maximum score indicating the most severe form [27,28].

Since ischemia-reperfusion injury produces severe motor deficits, therefore, the memory of animals was evaluated using the object recognition test, in which memory evaluation is independent of motor activity $[29,30]$. In this test, animals were subjected to 3 sessions including 'habituation' on day 0 (i.e., a day before surgery), 'familiarization' on day 1 (i.e., on a day of surgery, but performed before doing surgery) and 'test' on day 2 (i.e., 24 $\mathrm{h}$ after surgery, before sacrificing mice). In habituation, animals were kept in an arena for five minutes and in familiarization, 2 different objects were placed in the arena and mice explored those objects for $5 \mathrm{~min}$. On the test day, one of the objects was replaced with a new object and mice were allowed to explore those objects for $5 \mathrm{~min}$. Depending on the difference in time spent by mice in exploring new and the familiar object, the discrimination index was calculated by the following formula:

$$
\text { Discrimination index }=\left(\mathrm{T}_{\text {Novel }}-\mathrm{T}_{\text {Familiar }}\right) /\left(\mathrm{T}_{\text {Novel }}+\mathrm{T}_{\text {Familiar }}\right) \times 100 \%
$$

\section{Assessment of biochemical parameters in the brain homogenate}

After performing the behavioral tests, the animals were sacrificed by cervical dislocation to remove the brains. The brain was homogenized in the freshly prepared phosphate buffer $(\mathrm{pH}=7.4)$ and centrifuged to obtain the supernatant. The different biochemical tests were performed in the brain homogenate supernatant. The levels of p-STAT3 and connexin 43 were estimated in the brain homogenate using commercially available ELISA kits and estimations were done as per instructions of the kit.

\section{Assessment of cerebral infarction}

The mice brains were kept in refrigerator and thereafter, these were cut into thin slices. These were stained using triphenyl tetrazolium chloride (TTC) and cerebral infarction was calculated de- 
pending on the differential staining of infarcted and viable brain portions [31].

\section{Experimental design}

Seven groups $(n=8)$ were employed in this present study and details of these groups is explained below.

I. Normal: Animals were not subjected to any intervention and the different tests were performed in the normal mice.

II. Sham: The carotid arteries were exposed and thread was passed below the arteries, without any occlusion. Afterwards, the sutures were made to close the wound and animals were kept for $24 \mathrm{~h}$. The different behavioral and biochemical tests were performed in these animals before subjecting to surgery and $24 \mathrm{~h}$ after performing surgery.

III. Ischemia-reperfusion injury: After surgery, both the carotid arteries were occluded to induce global ischemia for $20 \mathrm{~min}$, which was followed by reperfusion for $24 \mathrm{~h}$ by de-occluding the carotid arteries. The different behavioral and biochemical tests were performed in these animals before subjecting to surgery and $24 \mathrm{~h}$ after performing surgery.

IV and V. Imatinib (20 and $40 \mathrm{mg} / \mathrm{kg}$ ) in ischemia-reperfusion injury: Two different doses of imatinib $(20 \mathrm{mg} / \mathrm{kg}$ i.p.) and (40 mg/kg i.p.) were administered in two different groups, 30 min prior to subjecting to ischemia-reperfusion injury. The different behavioral and biochemical tests were performed in these animals before subjecting to surgery and $24 \mathrm{~h}$ after performing surgery.

VI and VII. AG490 (3 and $6 \mathrm{mg} / \mathrm{kg})$ in imatinib $(40 \mathrm{mg} / \mathrm{kg})$ treated ischemia-reperfusion injury: AG490 (3 and $6 \mathrm{mg} / \mathrm{kg}$ i.p.) was administered $30 \mathrm{~min}$ prior to imatinib $(40 \mathrm{mg} / \mathrm{kg}$ i.p.) treatment in ischemia-reperfusion-subjected mice. The different behavioral and biochemical tests were performed in these animals before subjecting to surgery and $24 \mathrm{~h}$ after performing surgery.

\section{Statistical analysis}

The results of the study were denoted as mean \pm standard deviation. The data were analyzed using One way ANOVA followed by Bonferroni post hoc test. The value of $\mathrm{p}<0.05$ was considered to statistically significant.

\section{RESULTS}

\section{Impairment in locomotor activity, motor in- coordination, development of neurological deficits, impairment in memory and cerebral injury in global ischemia-reperfusion-subjected mice}

There was a marked reduction in the locomotor activity (decrease in score in an actophotometer test) (Fig. 1A); motor in- coordination (increase in score in the inclined beam walking test) (Fig. 1B), development of neurological deficits (increase in neurological severity score) (Fig. 2A) along with decease in learning and memory (decrease in discrimination index in object recognition test) (Fig. 3) following $20 \mathrm{~min}$ of global ischemia and $24 \mathrm{~h}$ of reperfusion injury. Moreover, global ischemia and reperfusion led to significant cerebral injury assessed in terms of increase in cerebral infarction. Indeed in ischemia-reperfusion subjected mice, there was marked increase in cerebral infarction assessed using TTC staining in comparison to sham control mice (Fig. 2B).

\section{Influence of pharmacological modulators on ischemia-reperfusion-induced alterations in behavioral parameters and cerebral infarction}

Administration of a single dose of imatinib $(20$ and $40 \mathrm{mg} /$ $\mathrm{kg}$ i.p.) led to dose-dependent improvement in behavioral altera-

\section{A}

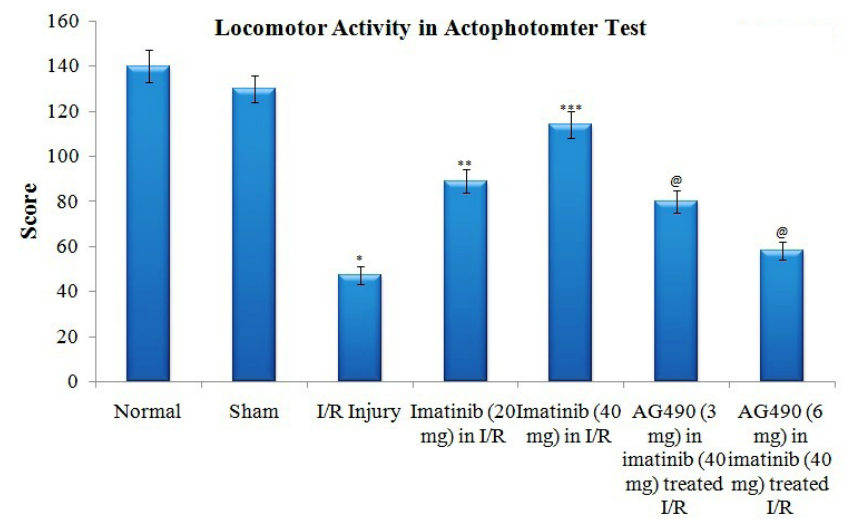

B

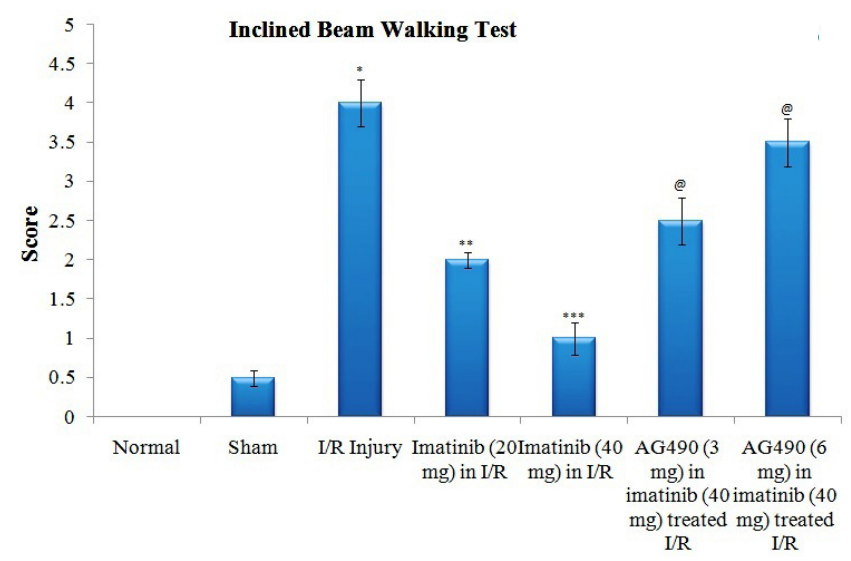

Fig. 1. Influence of global ischemia-reperfusion (I/R) injury and pharmacological modulators. (A) Locomotor activity assessed in the actophotometer test and (B) motor coordination assessed using inclined beam walking test. Values are represented in mean \pm standard deviation. ${ }^{*} p<0.05$ vs. normal; ${ }^{* *} p<0.05$ vs. ischemia-reperfusion I/ $\mathrm{R}$ injury; ${ }^{* * *} \mathrm{p}<0.05$ vs. imatinib $(20 \mathrm{mg} / \mathrm{kg})$ in $\mathrm{I} / \mathrm{R}$ injury; ${ }^{\circledR} \mathrm{p}<0.05$ vs. imatinib (40 mg/kg) in I/R injury. 


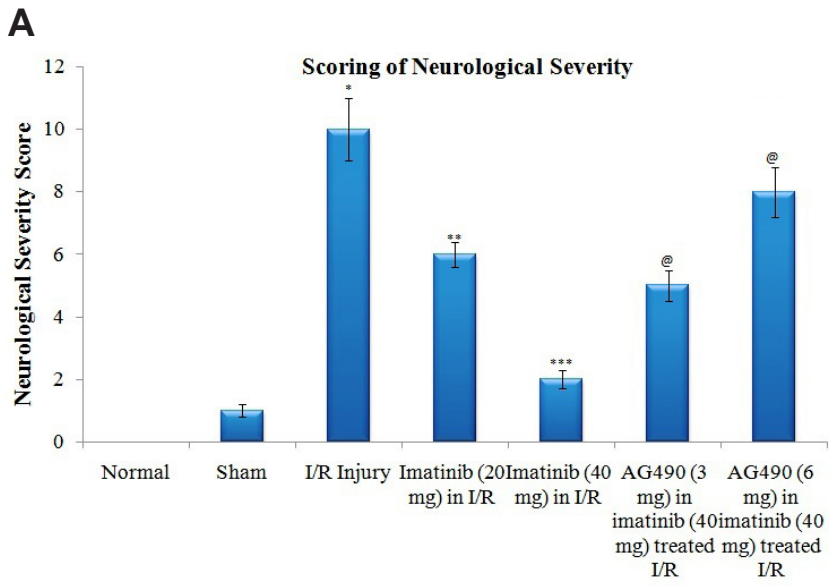

B

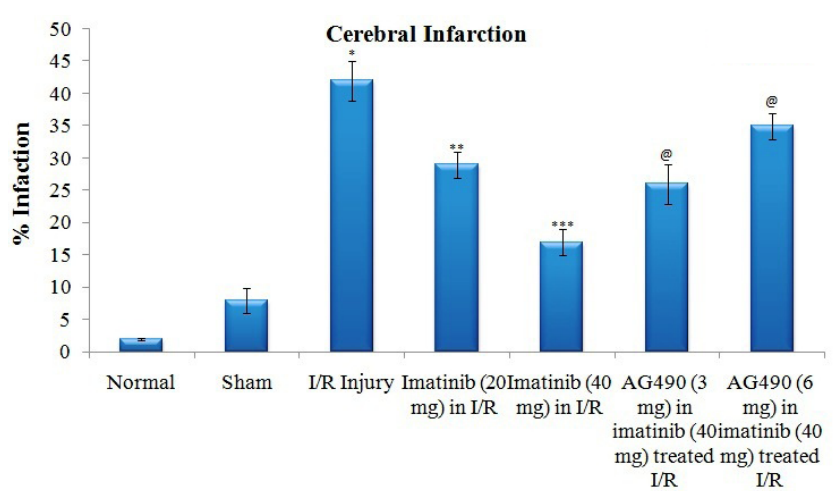

Fig. 2. Influence of global ischemia-reperfusion (I/R) injury and pharmacological modulators. (A) Neurological severity score and (B) cerebral infarction. Values are represented in mean \pm standard deviation. ${ }^{*} p<0.05$ vs. normal; ${ }^{* *} p<0.05$ vs. ischemia-reperfusion I/R injury; ${ }^{* * *} \mathrm{p}<0.05 \mathrm{vs}$. imatinib $(20 \mathrm{mg} / \mathrm{kg})$ in l/R injury; ${ }^{\circledR} p<0.05 \mathrm{vs}$. imatinib (40 $\mathrm{mg} / \mathrm{kg}$ ) in I/R injury.

tions induced due to global ischemia and reperfusion. There was an improvement in the locomotor activity, motor coordination, decrease in neurological severity score, increase in discrimination index (memory test) and decrease in cerebral infarction in imatinib-treated mice. However, prior administration of AG490 significantly abolished imatinib-mediated improvement in behavioral parameters in ischemia-reperfusion-subjected mice. The improvement in locomotor activity (Fig. 1A), motor coordination (Fig. 1B), neurological deficits (Fig. 2A), learning and memory (Fig. 3) imparted in the presence of imatinib was attenuated with the prior administration of AG490. Moreover, AG490 also attenuated imatinib-mediated decrease in cerebral infarction in global ischemia-reperfusion injury subjected mice (Fig. 2B).

\section{Changes in the biochemical parameters in the brain homogenate due to ischemia-reperfusion injury and pharmacological modulators}

There was a significant decrease in the expression of connexin

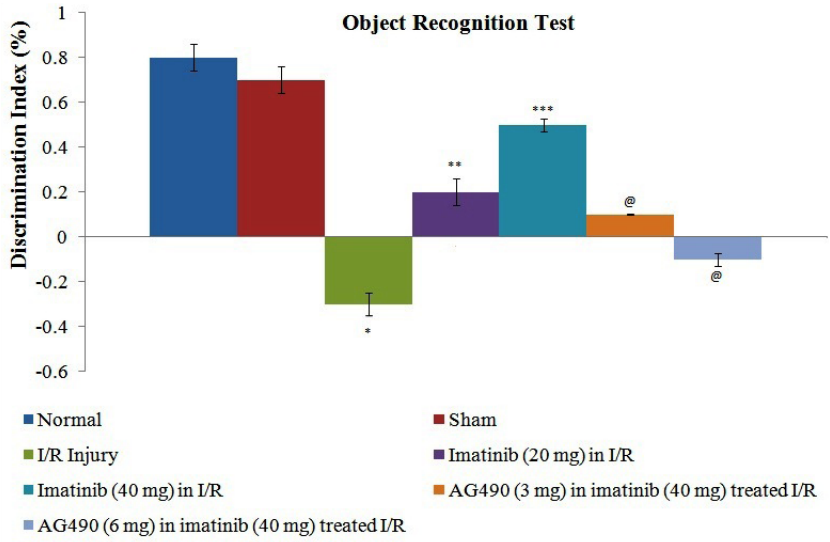

Fig. 3. Influence of global ischemia-reperfusion (I/R) injury and pharmacological modulators on discrimination index as a parameter of learning and memory assessed using object recognition test. Values are represented in mean \pm standard deviation. ${ }^{*} p<0.05$ vs. normal; ${ }^{* *} \mathrm{p}<0.05 \mathrm{vs}$. I/R injury; ${ }^{* * *} \mathrm{p}<0.05 \mathrm{vs}$. imatinib $(20 \mathrm{mg} / \mathrm{kg})$ in l/ R injury; ${ }^{\circledR} p<0.05$ vs. imatinib (40 mg/kg) in l/R injury.

43 and p-STAT3 in the brain homogenate following $20 \mathrm{~min}$ of global ischemia and $24 \mathrm{~h}$ of reperfusion. However, imatinibmediated improvement in behavioral parameters and decrease in cerebral infarction was also associated with the normalization of biochemical alterations in ischemia-reperfusion-subjected mice. Treatment with imatinib (20 and $40 \mathrm{mg} / \mathrm{kg}$ ) led to normalization of connexin 43 and p-STAT3 expression in the brain. However, prior administration of AG490 attenuated the effects of imatinib on the biochemical parameters and there was significant decrease in the expression of connexin 43 (Fig. 4A) and p-STAT3 (Fig. 4B) in AG490 administered mice.

\section{DISCUSSION}

Since there is a significant influence of cyclical variations of circulating estrogen levels on the cerebral ischemia-reperfusion injury [32,33], therefore, male mice were employed in the present study. In this study, 20 min of global cerebral ischemia and $24 \mathrm{~h}$ of reperfusion led to significant brain injury, assessed in terms of decrease in the locomotor activity, development of motor incoordination and neurological deficits. Moreover, there was a significant decline in learning and memory in ischemia-reperfusionsubjected mice assessed by measuring discrimination index using a discrimination test. The assessment of learning and memory using a discrimination test is advantageous as this test is minimally dependent on the locomotor activity, which is in contrast to typical maze tests in which assessment of memory is dependent on locomotor activity $[34,35]$. Due to this, discrimination-based memory test is particularly useful in situations in which there is a locomotor impairment such as in this present study. Apart from these, global ischemia and reperfusion also led to increase in the 
A

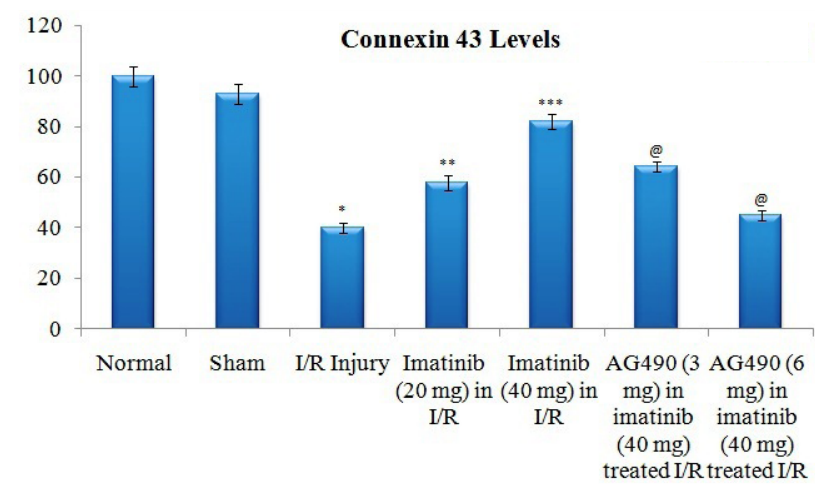

B

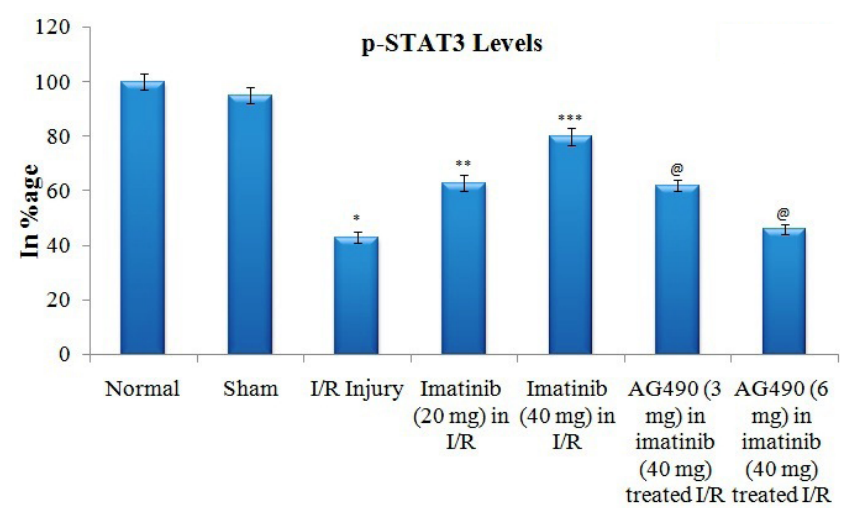

Fig. 4. Influence of global ischemia-reperfusion (I/R) injury and pharmacological modulators. (A) Connexin 43 levels and (B) phosphorylated signal transducer and activator of transcription 3 ( $p$-STAT3) levels in the brain homogenate. Values are represented in mean \pm standard deviation. ${ }^{*} p<0.05$ vs. normal; ${ }^{* *} p<0.05$ vs. l/R injury; ${ }^{* * *} p<0.05$ vs. imatinib $(20 \mathrm{mg} / \mathrm{kg})$ in $\mathrm{l} / \mathrm{R}$ injury; ${ }^{\circledR} \mathrm{p}<0.05 \mathrm{vs}$. imatinib $(40 \mathrm{mg} / \mathrm{kg})$ in I/R injury.

cerebral infarction, assessed using TTC staining (Fig. 5).

In the present investigation, treatment with a single dose of imatinib (20 and $40 \mathrm{mg} / \mathrm{kg}$ ) significantly attenuated global ischemia-reperfusion injury-induced cerebral injury in terms of improvement in behavioral parameters, normalization of learning and memory along with decrease in cerebral infarction. Imatinib is an anticancer drug and it has been used in different cancers including chronic myeloid leukemia and gastrointestinal stromal tumor $[1,2]$. Furthermore, preclinical and clinical studies have shown the beneficial role of imatinib in the different diseases affecting the brain including Alzheimer disease, Parkinson disease and multiple sclerosis $[3,36]$. Moreover, its efficacy in attenuating acute ischemic stroke [6], tissue Plasminogen activator [7], subarachnoid hemorrhage [9], intracerebral hemorrhage [10] and traumatic brain injury [8]-induced blood brain barrier disruption has also been reported. However to best of our knowledge, it is the first research study depicting the potential of imatinib in attenuating global ischemia-reperfusion-induced cerebral infarction, decline in memory along with behavioral and neurological
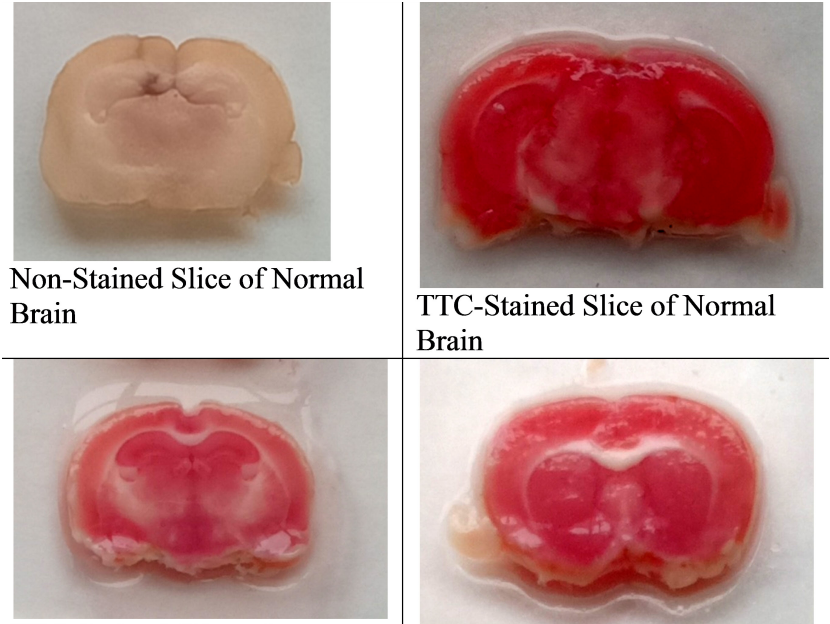

TTC-Stained Slice of

Ischemia-Reperfusion

Subjected Brain

Brain

Fig. 5. Representaive pictures of TTC-stained brain slices for determining the extent of cerebral infarction. TTC, triphenyl tetrazolium chloride.

deficits.

In the present study, there was a significant decrease in the levels of p-STAT3 and connexin 43 in the brains of the mice subjected to $20 \mathrm{~min}$ of global ischemia and $24 \mathrm{~h}$ of reperfusion. STAT3 is a transcriptional factor and it belongs to the family of STAT proteins. Activation of JAK2 leads to the phosphorylation of STAT3, which in turn is translocated in the nucleus to influence the protein expression. There have been previous studies showing a decrease in the levels of p-STAT3 in the brain in response to ischemia-reperfusion injury and increase in the expression of p-STAT3 is associated with neuroprotective effects $[37,38]$. Studies have shown that the increase in the expression of STAT3, a transcriptional factor, is associated with a decrease in inflammatory mediators [39]. Indeed, the actions of anti-inflammatory cytokine (interleukin-10) are mediated through activation of JAK2/ STAT3 pathway [40,41]. Connexin 43 is a gap junction protein and it forms the structural unit of gap junctions, predominantly in the central nervous system. Studies have shown that connexin 43-dependent gap junctional channels play an important role in maintaining the communication amongst different neurons and hence, these are critical in maintaining brain homeostasis [42]. Moreover, studies have shown the protective role of connexin 43 and it has been projected as a key therapeutic target to confer tissue protection $[19,43,44]$. It is reported that connexin 43 produces neuroprotection by virtue of multiple effects including decreased release of pro-inflammatory factors from microglial cells [45] and maintaining the functional status of mitochondria [46]. Indeed, mice lacking connexin 43 in astrocytes exhibit an increase in apoptosis and neuroinflammation following cerebral ischemia [47].

In this study, treatment with a single dose of imatinib signifi- 
cantly attenuated ischemia-reperfusion-induced decrease in the levels of p-STAT3 and connexin 43 in the brain. Accordingly, it may be suggested that imatinib-mediated beneficial effects are possibly mediated through activation of p-STAT3 and connexin 43 signaling pathway. The relationship between imatinib and $\mathrm{p}$ STAT3 was further established by the results of this study showing that the beneficial effects of imatinib were significantly attenuated in the presence of AG490, a selective JAK-STAT3 inhibitor. Administration of AG490 significantly attenuated the beneficial effects of imatinib in global ischemia-reperfusion-induced behavioral changes, neurological deficits, cerebral infarction and decline in memory. It suggests the importance of JAK-STAT3 signaling pathway activation in triggering neuroprotection in response to imatinib treatment. There have been previous studies showing that the activation of JAK2/STAT3 signaling pathway triggers neuroprotection in different models $[14,15]$.

Apart from attenuating the beneficial effects of imatinib, administration of AG490 also attenuated the levels of p-STAT3 and connexin 43 in imatinib-treated mice. The decrease in the p-STAT3 levels in the presence of AG490 signifies that JAK2 activation is responsible for increasing the levels of phosphorylated form of STAT3. The decrease in the levels of connexin 43 in the presence of AG490 signifies that the expression of connexin 43 is dependent on the activation of JAK-STAT3 pathway and connexin 43 is a downstream mediator of JAK-STAT3 signaling pathway. A previous study had also demonstrated that an increase in connexin 43 is dependent on the activation of JAK/STAT pathway [48]. Based on this, it may be proposed that imatinib-mediated neuroprotective effects in global ischemia reperfusion injury may involve activation of the JAK2/STAT3 signaling pathway along with the increase in the expression of connexin 43 (Fig. 6).

It is an interesting finding that imatinib, being a tyrosine kinase inhibitor, promoted the activation of JAK2/STAT3 pathway and increased the levels of phosphorylated form of STAT3. However, the results of the present study are not sufficient enough to delineate the precise mechanisms responsible for increase in the phosphorylated form of STAT3 and activation of JAK2 pathway in the presence of imatinib. The activation of JAK2/STAT3 pathway is linked to multiple pathways including glycogen synthase kinase (GSK)-3 $3[49,50]$. It is also reported that imatinib decreases the phosphorylation of GSK-3 $\beta$ and increases the enzyme activity of GSK-3 $\beta$ [51,52]. Moreover, there has been a report suggesting that the activation of JAK2 is negatively regulated by a scaffolding protein 'ninein' [53], which in turn is phosphorylated in the presence of GSK-3 $\beta$ [54]. Accordingly, it may be hypothesized that imatinib-mediated decrease in phosphorylation of GSK-3 $\beta$ and increase in GSK-3 $\beta$ activity may activate the JAK2 pathway leading to increase in the phosphorylation of STAT3. However, future studies are required to experimentally verify the above described hypothesis that imatinib may activate the GSK- $3 \beta$ activity to trigger the activation of JAK2/STAT3 signaling pathway in inducing neuroprotection in cerebral ischemia-reperfusion injury model.

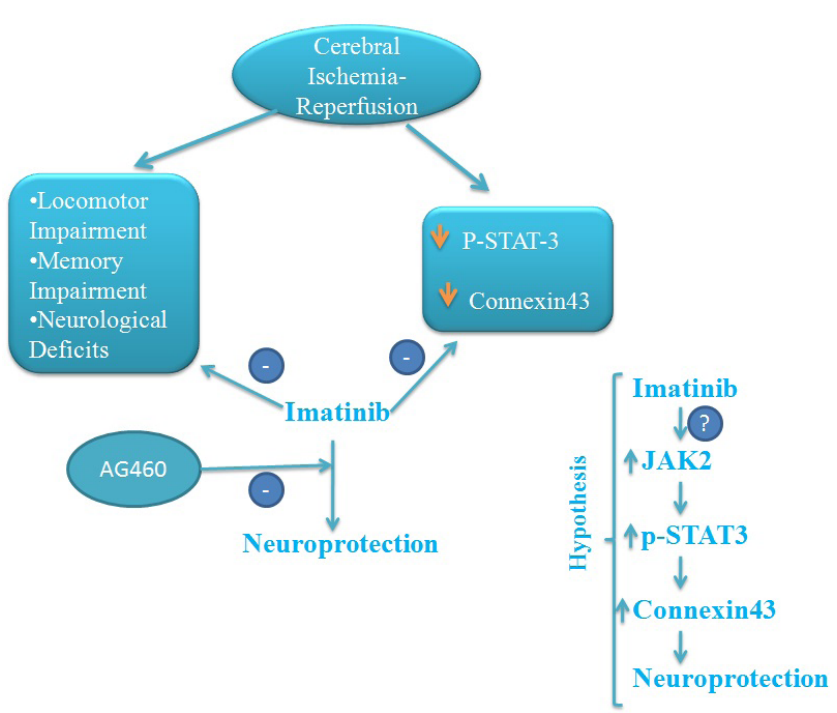

Fig. 6. Proposed hypothesis of imatinib-induced neuroprotection in cerebral ischemia-reperfusion induced injury. p-STAT3, phosphorylated signal transducer and activator of transcription 3; JAK2, Janus-activated kinase 2.

Imatinib has the therapeutic potential in attenuating global ischemia reperfusion-induced cerebral injury, which may be possibly attributed to activation of JAK2/STAT3 signaling pathway along with the increase in the expression of connexin 43.

\section{CONFLICTS OF INTEREST}

The authors declare no conflicts of interest.

\section{REFERENCES}

1. Baccarani M, Saglio G, Goldman J, Hochhaus A, Simonsson B, Appelbaum F, Apperley J, Cervantes F, Cortes J, Deininger M, Gratwohl A, Guilhot F, Horowitz M, Hughes T, Kantarjian H, Larson R, Niederwieser D, Silver R, Hehlmann R, European LeukemiaNet. Evolving concepts in the management of chronic myeloid leukemia: recommendations from an expert panel on behalf of the European LeukemiaNet. Blood. 2006;108:1809-1820.

2. Ben Ami E, Demetri GD. A safety evaluation of imatinib mesylate in the treatment of gastrointestinal stromal tumor. Expert Opin Drug Saf. 2016;15:571-578.

3. Kumar M, Kulshrestha R, Singh N, Jaggi AS. Expanding spectrum of anticancer drug, imatinib, in the disorders affecting brain and spinal cord. Pharmacol Res. 2019;143:86-96.

4. Wu R, Chen H, Ma J, He Q, Huang Q, Liu Q, Li M, Yuan Z. c-Ablp38 $\alpha$ signaling plays an important role in MPTP-induced neuronal death. Cell Death Differ. 2016;23:542-552.

5. Cancino GI, Perez de Arce K, Castro PU, Toledo EM, von Bernhardi $\mathrm{R}$, Alvarez AR. c-Abl tyrosine kinase modulates tau pathology and Cdk5 phosphorylation in AD transgenic mice. Neurobiol Aging. 2011;32:1249-1261. 
6. Merali Z, Leung J, Mikulis D, Silver F, Kassner A. Longitudinal assessment of imatinib's effect on the blood-brain barrier after ischemia/reperfusion injury with permeability MRI. Transl Stroke Res. 2015;6:39-49.

7. Su EJ, Fredriksson L, Geyer M, Folestad E, Cale J, Andrae J, Gao Y, Pietras K, Mann K, Yepes M, Strickland DK, Betsholtz C, Eriksson $\mathrm{U}$, Lawrence DA. Activation of PDGF-CC by tissue plasminogen activator impairs blood-brain barrier integrity during ischemic stroke. Nat Med. 2008;14:731-737.

8. Su EJ, Fredriksson L, Kanzawa M, Moore S, Folestad E, Stevenson TK, Nilsson I, Sashindranath M, Schielke GP, Warnock M, Ragsdale M, Mann K, Lawrence AL, Medcalf RL, Eriksson U, Murphy GG, Lawrence DA. Imatinib treatment reduces brain injury in a murine model of traumatic brain injury. Front Cell Neurosci. 2015;9:385.

9. Zhan Y, Krafft PR, Lekic T, Ma Q, Souvenir R, Zhang JH, Tang J. Imatinib preserves blood-brain barrier integrity following experimental subarachnoid hemorrhage in rats. J Neurosci Res. 2015;93:94-103.

10. Ma Q, Huang B, Khatibi N, Rolland W 2nd, Suzuki H, Zhang JH, Tang J. PDGFR- $\alpha$ inhibition preserves blood-brain barrier after intracerebral hemorrhage. Ann Neurol. 2011;70:920-931.

11. Harukuni I, Bhardwaj A. Mechanisms of brain injury after global cerebral ischemia. Neurol Clin. 2006;24:1-21.

12. Wiklund L, Martijn C, Miclescu A, Semenas E, Rubertsson S, Sharma HS. Central nervous tissue damage after hypoxia and reperfusion in conjunction with cardiac arrest and cardiopulmonary resuscitation: mechanisms of action and possibilities for mitigation. Int Rev Neurobiol. 2012;102:173-187.

13. Tang Y, Tong X, Li Y, Jiang G, Yu M, Chen Y, Dong S. JAK2/STAT3 pathway is involved in the protective effects of epidermal growth factor receptor activation against cerebral ischemia/reperfusion injury in rats. Neurosci Lett. 2018;662:219-226.

14. Li Y, Zhang X, Cui L, Chen R, Zhang Y, Zhang C, Zhu X, He T, Shen Z, Dong L, Zhao J, Wen Y, Zheng X, Li P. Salvianolic acids enhance cerebral angiogenesis and neurological recovery by activating JAK2/ STAT3 signaling pathway after ischemic stroke in mice. J Neurochem. 2017;143:87-99.

15. Chen M, Zou W, Chen M, Cao L, Ding J, Xiao W, Hu G. Ginkgolide $\mathrm{K}$ promotes angiogenesis in a middle cerebral artery occlusion mouse model via activating JAK2/STAT3 pathway. Eur J Pharmacol. 2018;833:221-229.

16. Xing L, Yang T, Cui S, Chen G. Connexin hemichannels in astrocytes: role in CNS disorders. Front Mol Neurosci. 2019;12:23.

17. Liu WJ, Yang J. Preferentially regulated expression of connexin 43 in the developing spiral ganglion neurons and afferent terminals in post-natal rat cochlea. Eur J Histochem. 2015;59:2464.

18. Wu LY, Yu XL, Feng LY. Connexin 43 stabilizes astrocytes in a stroke-like milieu to facilitate neuronal recovery. Acta Pharmacol Sin. 2015;36:928-938.

19. Schulz R, Görge PM, Görbe A, Ferdinandy P, Lampe PD, Leybaert L. Connexin 43 is an emerging therapeutic target in ischemia/reperfusion injury, cardioprotection and neuroprotection. Pharmacol Ther. 2015;153:90-106.

20. Yi C, Koulakoff A, Giaume C. Astroglial connexins as a therapeutic target for Alzheimer's disease. Curr Pharm Des. 2017;23:4958-4968.

21. Weintraub MK, Bisson CM, Nouri JN, Vinson BT, Eimerbrink MJ, Kranjac D, Boehm GW, Chumley MJ. Imatinib methanesulfonate reduces hippocampal amyloid- $\beta$ and restores cognitive function following repeated endotoxin exposure. Brain Behav Immun. 2013;33:24-28.

22. Cancino GI, Toledo EM, Leal NR, Hernandez DE, Yévenes LF, Inestrosa NC, Alvarez AR. STI571 prevents apoptosis, tau phosphorylation and behavioural impairments induced by Alzheimer's betaamyloid deposits. Brain. 2008;131(Pt 9):2425-2442.

23. Chu J, Lauretti E, Craige CP, Praticò D. Pharmacological modulation of GSAP reduces amyloid- $\beta$ levels and tau phosphorylation in a mouse model of Alzheimer's disease with plaques and tangles. $J$ Alzheimers Dis. 2014;41:729-737.

24. Chen B, Yang L, Chen J, Chen Y, Zhang L, Wang L, Li X, Li Y, Yu H. Inhibition of Connexin43 hemichannels with Gap19 protects cerebral ischemia/reperfusion injury via the JAK2/STAT3 pathway in mice. Brain Res Bull. 2019;146:124-135.

25. Kim SJ, Lee SR. Protective effect of melatonin against transient global cerebral ischemia-induced neuronal cell damage via inhibition of matrix metalloproteinase-9. Life Sci. 2014;94:8-16.

26. He JT, Li H, Yang L, Cheng KL. Involvement of endothelin-1, H2S and Nrf2 in beneficial effects of remote ischemic preconditioning in global cerebral ischemia-induced vascular dementia in mice. Cell Mol Neurobiol. 2019;39:671-686.

27. Rodriguez R, Santiago-Mejia J, Gomez C, San-Juan ER. A simplified procedure for the quantitative measurement of neurological deficits after forebrain ischemia in mice. J Neurosci Methods. 2005;147:2228.

28. Ya BL, Li HF, Wang HY, Wu F, Xin Q, Cheng HJ, Li WJ, Lin N, Ba ZH, Zhang RJ, Liu Q, Li YN, Bai B, Ge F. 5-HMF attenuates striatum oxidative damage via Nrf2/ARE signaling pathway following transient global cerebral ischemia. Cell Stress Chaperones. 2017;22:55-65.

29. Leger M, Quiedeville A, Bouet V, Haelewyn B, Boulouard M, Schumann-Bard P, Freret T. Object recognition test in mice. Nat Protoc. 2013;8:2531-2537.

30. Yang Y, Lu F, Zhuang L, Yang S, Kong Y, Tan W, Gong Z, Zhan S. Combined preconditioning with hypoxia and GYKI-52466 protects rats from cerebral ischemic injury by HIF-1 $\alpha /$ eNOS pathway. Am $J$ Transl Res. 2017;9:5308-5319.

31. Okuno S, Nakase H, Sakaki T. Comparative study of 2,3,5-triphenyltetrazolium chloride (TTC) and hematoxylin-eosin staining for quantification of early brain ischemic injury in cats. Neurol Res. 2001;23:657-661.

32. Suzuki S, Brown CM, Wise PM. Neuroprotective effects of estrogens following ischemic stroke. Front Neuroendocrinol. 2009;30:201-211.

33. Bi R, Foy MR, Vouimba RM, Thompson RF, Baudry M. Cyclic changes in estradiol regulate synaptic plasticity through the MAP kinase pathway. Proc Natl Acad Sci U S A. 2001;98:13391-13395.

34. Antunes M, Biala G. The novel object recognition memory: neurobiology, test procedure, and its modifications. Cogn Process. 2012;13:93-110.

35. Lueptow LM. Novel object recognition test for the investigation of learning and memory in mice. J Vis Exp. 2017;(126):55718.

36. Estrada LD, Chamorro D, Yañez MJ, Gonzalez M, Leal N, von Bernhardi R, Dulcey AE, Marugan J, Ferrer M, Soto C, Zanlungo S, Inestrosa $\mathrm{NC}$, Alvarez AR. Reduction of blood amyloid- $\beta$ oligomers in Alzheimer's disease transgenic mice by c-Abl kinase inhibition. $J$ Alzheimers Dis. 2016;54:1193-1205. 
37. Zhao Y, Xue Y, Liu Z, Ren S, Guan X, Li M, Zhao X, Song Y, Ren $X$. Role of the Janus kinase 2/signal transducers and activators of transcription 3 pathway in the protective effect of remote ischemia preconditioning against cerebral ischemia-reperfusion injury in rats. Neuroreport. 2019;30:664-670.

38. Jung JE, Kim GS, Chan PH. Neuroprotection by interleukin-6 is mediated by signal transducer and activator of transcription 3 and antioxidative signaling in ischemic stroke. Stroke. 2011;42:35743579.

39. Tu S, Xiao F, Min X, Chen H, Fan X, Cao K. Catechin attenuates coronary heart disease in a rat model by inhibiting inflammation. Cardiovasc Toxicol. 2018;18:393-399.

40. Zhu Y, Liu Z, Peng YP, Qiu YH. Interleukin-10 inhibits neuroinflammation-mediated apoptosis of ventral mesencephalic neurons via JAK-STAT3 pathway. Int Immunopharmacol. 2017;50:353-360.

41. Sharma S, Yang B, Xi X, Grotta JC, Aronowski J, Savitz SI. IL-10 directly protects cortical neurons by activating PI-3 kinase and STAT3 pathways. Brain Res. 2011;1373:189-194.

42. Vicario N, Zappalà A, Calabrese G, Gulino R, Parenti C, Gulisano M, Parenti R. Connexins in the central nervous system: physiological traits and neuroprotective targets. Front Physiol. 2017;8:1060.

43. Hanstein R, Trotter J, Behl C, Clement AB. Increased connexin 43 expression as a potential mediator of the neuroprotective activity of the corticotropin-releasing hormone. Mol Endocrinol. 2009;23:14791493.

44. Chen YS, Green CR, Teague R, Perrett J, Danesh-Meyer HV, Toth I, Rupenthal ID. Intravitreal injection of lipoamino acid-modified connexin43 mimetic peptide enhances neuroprotection after retinal ischemia. Drug Deliv Transl Res. 2015;5:480-488.

45. Ma Y, Bu J, Dang H, Sha J, Jing Y, Shan-jiang AI, Li H, Zhu Y. Inhibition of adenosine monophosphate-activated protein kinase reduces glial cell-mediated inflammation and induces the expression of Cx43 in astroglias after cerebral ischemia. Brain Res. 2015;1605:111.

46. Kim IS, Ganesan P, Choi DK. Cx43 mediates resistance against
$\mathrm{MPP}^{+}$-induced apoptosis in SH-SY5Y neuroblastoma cells via modulating the mitochondrial apoptosis pathway. Int J Mol Sci. 2016;17:E1819.

47. Nakase T, Söhl G, Theis M, Willecke K, Naus CC. Increased apoptosis and inflammation after focal brain ischemia in mice lacking connexin43 in astrocytes. Am J Pathol. 2004;164:2067-2075.

48. Ozog MA, Bernier SM, Bates DC, Chatterjee B, Lo CW, Naus CC. The complex of ciliary neurotrophic factor-ciliary neurotrophic factor receptor alpha up-regulates connexin 43 and intercellular coupling in astrocytes via the Janus tyrosine kinase/signal transducer and activator of transcription pathway. Mol Biol Cell. 2004;15:47614774.

49. Gao S, Li S, Duan X, Gu Z, Ma Z, Yuan X, Feng X, Wang H. Inhibition of glycogen synthase kinase 3 beta (GSK3 $\beta$ ) suppresses the progression of esophageal squamous cell carcinoma by modifying STAT3 activity. Mol Carcinog. 2017;56:2301-2316.

50. Beurel E, Jope RS. Differential regulation of STAT family members by glycogen synthase kinase-3. J Biol Chem. 2008;283:21934-21944.

51. Reddiconto G, Toto C, Palamà I, De Leo S, de Luca E, De Matteis S, Dini L, Passerini CG, Di Renzo N, Maffia M, Coluccia AM. Targeting of GSK3 $\beta$ promotes imatinib-mediated apoptosis in quiescent CD34+ chronic myeloid leukemia progenitors, preserving normal stem cells. Blood. 2012;119:2335-2345.

52. Niu CC, Zhao C, Yang ZD, Zhang XL, Wu WR, Pan J, Zhao C, Li ZQ, Ding W, Yang Z, Si WK. Downregulation of $\gamma$-catenin inhibits CML cell growth and potentiates the response of CML cells to imatinib through $\beta$-catenin inhibition. Int J Mol Med. 2013;31:453-458.

53. Jay J, Hammer A, Nestor-Kalinoski A, Diakonova M. JAK2 tyrosine kinase phosphorylates and is negatively regulated by centrosomal protein Ninein. Mol Cell Biol. 2015;35:111-131.

54. Howng SL, Hsu HC, Cheng TS, Lee YL, Chang LK, Lu PJ, Hong YR. A novel ninein-interaction protein, CGI-99, blocks ninein phosphorylation by GSK3beta and is highly expressed in brain tumors. FEBS Lett. 2004;566:162-168. 